Monatsschr Kinderheilkd $2013 \cdot$ 161:389-389

DOI 10.1007/s00112-012-2796-0

Online publiziert: 1. Mai 2013

○) Springer-Verlag Berlin Heidelberg 2013

\title{
G. Hansen
}

Klinik für Pädiatrische Pneumologie, Allergologie und Neonatologie,

Medizinische Hochschule Hannover, Carl-Neuberg-Str. 1

\section{Chronische Atemwegserkrankungen}

Deutlich seltener als die Mukoviszidose ist die primäre ziliäre Dyskinesie, eine angeborene seltene Störung der Zilienmotilität, die sich v. a. im Respirationstrakt manifestiert. Eine frühzeitige Diagnosestellung ist für die Betroffenen wichtig, um so eine unnötige und belastende Suche nach anderen Krankheitsursachen $\mathrm{zu}$ vermeiden und eine fokussierte Therapie des Erkrankungsverlaufes frühzeitig zu beginnen. Heymut Omran et al. stellen eine Diagnostikempfehlung vor, die praktische Hinweise für das richtige Vorgehen bei Verdacht auf eine primäre ziliäre Dyskinesie anbietet.

In Zukunft werden uns viel häufiger Kinder mit einer bronchopulmonalen Dysplasie (BPD) begegnen, einer Multisystemerkrankung bei kleinen Frühgeborenen. Durch die zunehmend höhere Überlebenschance sehr kleiner Frühgeborener wird diese Erkrankung immer mehr Kinder betreffen. Eine genaue Kenntnis der Erkrankung, der Möglichkeiten zur Prävention und zur Therapie wird deshalb immer wichtiger für den Kinderarzt werden. Egbert Herting fasst in seinem Artikel zur BPD den aktuellen Stand der therapeutischen Möglichkeiten zusammen.

Wir hoffen, dass wir Ihnen mit diesen Beiträgen zu wesentlichen chronischen Erkrankungen der Lunge bei Kindern nützliche Informationen für Ihren klinischen Alltag anbieten und wünschen Ihnen interessante und neue Erkenntnisse beim Lesen!

Ihre

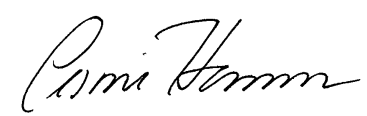

Prof. Dr. med. Gesine Hansen

\section{Korrespondenzadresse}

\author{
G. Hansen \\ Klinik für Pädiatrische Pneumologie, \\ Allergologie und Neonatologie, Medizinische \\ Hochschule Hannover \\ Carl-Neuberg-Str. 1 \\ 30625 Hannover \\ Hansen.Gesine@mh-hannover.de
}

Dank der zahlreichen Verbesserungen in der symptomatischen Therapie haben sich Lebenserwartung und -qualität bei CF-Patienten in den letzten Jahrzehnten stark verbessert. Erst kürzlich wurde mit der ersten klinischen Anwendung eines Chloridkanalpotentiators erstmals eine kausale Therapie erfolgreich durchgeführt. Burkhard Tümmler stellt Ihnen in seinem Artikel die Grundlagen und klinisch relevanten Aspekte dieser schweren Erkrankung dar. 